\title{
On the Translation of Children's Literature Based on Eco-Translatology: Taking Ren Rongrong's Translated Version of The Wind in the Willows as an Example
}

\author{
Yue Wang \\ School of Languages and Communication Studies, Beijing Jiaotong University, Beijing, China \\ Email: 19121764@bjtu.edu.cn
}

How to cite this paper: Wang, Y. (2021) On the Translation of Children's Literature Based on Eco-Translatology: Taking Ren Rongrong's Translated Version of The Wind in the Willows as an Example. Open Access Library Journal, 8: e7835.

https://doi.org/10.4236/oalib.1107835

Received: August 5, 2021

Accepted: October 26, 2021

Published: October 29, 2021

Copyright () 2021 by author(s) and Open Access Library Inc.

This work is licensed under the Creative Commons Attribution International License (CC BY 4.0).

http://creativecommons.org/licenses/by/4.0/

(c) (i) Open Access

\begin{abstract}
Compared with western children's literature, the development of Chinese local children's literature starts late, and there are still several problems such as lack of originality and low degree of internationalization. In order to enrich children's reading experiences, to promote their early emergent literacy and future academic achievements, introducing the classics of foreign children's literature is an effective way. In this process, translation serves as a bridge between the original text and the translation. At present, the literature focusing on the evaluation and analysis of children's literature translation is mainly based on those traditional translation theories such as reception aesthetics theory and functional equivalence theory, and there is still a lack of researches based on eco-translatology. Therefore, this paper is mainly devoted to sorting out the relevant tenets of eco-translatology studies, and taking this as the theoretical framework to comment on Ren Rongrong's translated version of The Wind in the Willows. On the one hand, this paper aims to evaluate the translation of children's literature based on eco-translatology. On the other hand, it analyzes the feasibility of eco-translatology when applying in analyzing the translated version of certain children's literature works.
\end{abstract}

\section{Subject Areas \\ Linguistics, Literature}

\section{Keywords}

Eco-Translatology, Children's Literature, The Wind in the Willows, English-Chinese Translation 


\section{1. 引言}

儿童文学因其形象生动活泼、语言通俗易懂而受到儿童读者的喜爱, 既 是儿童早期阅读的主要知识载体, 也是培养其早期读写能力的重要途径。除 了充当知识传递的媒介以外, 儿童文学为儿童读者提供了充足的语言符号, 使得儿童能够在脑中建构起可感知的、立体的文学形象, 这对于培养儿童的 审美感受力、判断力及理解力十分重要 [1]。同时, 儿童文学能够在情感上激 起并满足儿童的好奇心与求知欲, 达到儿童教育自己、完善自身行为的目的 [2]。西方儿童文学在儿童文学发展史上成果显著。但我国本土儿童文学起步 较晚, 目前仍存在原创性不足、国际化程度较低等问题 [3]。因此, 翻译成为 沟通国内外儿童文学发展的重要桥梁。

目前, 国内对于儿童文学翻译的研究主要停留在目的论、接受美学理论 等传统翻译理论的应用, 基于生态翻译学的研究鲜有涉及。生态翻译学的发 展兴起于 2001 年, 并于 2009 年前后全面展开。新生态主义翻译观的提出, 简化了生态翻译学理论体系的立论基础, 并澄清了既往理论中的质疑和误解: 它以达尔文 “生物进化论” 为基础, 吸纳了孔孟学说有关生态的哲学思想与 论述, 顺应了社会文明与现代哲学思想转型的要求, 蕴含着丰富的中国古代 哲学思想及生态智慧 [4], 不仅是本土译学研究的突破性与创新性成果 [5], 更 是西方生态主义与东方生态智慧的整合与集成[6]。该理论创造性地阐述了包 括生态理性、生态翻译环境等在内的九大研究焦点及理论视角 [7]。近年来,

国际上有关生态翻译学的讨论与研究也于近年来被推向了新的高度, 展示出 该理论高度的可行性与可推广性, 相关研究主要围绕翻译史、翻译批评研究、 翻译伦理研究、译学流派研究等方面开展 [8]。

因此, 本文旨在选择生态翻译学作为理论基础, 通过介绍其中有关 “译 有所为”、“译者中心性”、“适应性选择” 与 “选择性适应” 等理论内涵, 并运用 “三维转换” 理论作为研究方法展开对《柳林风声》[9]任溶溶译本的 讨论。一方面, 旨在通过生态翻译学来检验并评析任译本; 另一方面, 则对 生态翻译学在儿童文学翻译中所发挥的效力及作用进行分析。

\section{2. 基于生态翻译学的讨论}

作为英国爱德华时期最重要的儿童文学代表作之一, 《柳林风声》的问 世对 20 世纪英国幻想文学产生了深刻的影响, 也是英国儿童文学第一个黄金 时代后期的经典之作 [10], 被称作是 “英国散文体作品的典范” 。书中, 主人 公形象均依托于动物而存在, 但是它们身上却拥有一定的社会属性及人格特 征, 如: 思想上渴求自由与改变, 无惧险阻敢于冒险; 生活中需要刻意地保 持社交礼仪, 以期达到 “人际关系” 的平衡等。正是这种共通性, 使得书中 塑造的主人公形象跃然纸上。该书中有关自然生态的描写十分丰富, 具有高 度的艺术审美色彩, 阅读起来格外吸引眼球。童话故事给予了儿童想象力与 好奇心的生长空间, 他们可以搭造建构出属于自己的童话世界。该书从童话 幻想艺术的角度展示了少年儿童心向往之的理想生活: 他们不甘平庸、追求 刺激, 在面对新鲜事物时仍心怀期许, 并且勇于试误, 在不断的尝试中与身 
边人乃至周遭世界进行互动[11], 具有深刻的教育意义。在《柳林风声》任译 本中, 既体现出 “译者中心性” 的指导作用, 同时又体现出译者对于翻译生 态环境、翻译文本和翻译策略的选择与适应。因此, 本文旨在选择生态翻译 学作为理论基础, 通过介绍其中有关 “译有所为”、“译者中心性”、“适 应性选择” 与 “选择性适应” 等理论内涵, 简析任译作品风格, 并运用 “三 维转换” 理论作为研究方法展开对 《柳林风声》任译本的讨论, 以期发现任 译本的优势与不足, 是生态翻译学应用于儿童文学研究有效性的检验与发展。

\section{1. 任译作品风格简析}

任溶溶老师在进行作品译介时, 他能够时刻考虑到儿童的旨趣与发展特 征, 并以此来调整翻译策略, 是 “译者中心性” 的体现, 也为其译作提供了

“译有所为” 掌控下的支持。任溶溶老师身兼多重身份: 他既是翻译家, 又 是精通儿童文学写作的作家, 同时还是一名编辑。深厚的语言基础使得他的 译作与原创作品读起来通畅晓达、朗朗上口, 同时又贴近儿童生活日常, 符 合儿童阅读兴趣与习惯, 体现出自然、亲切、风趣、幽默的特点 [12]。随着翻 译与写作实践的积累, 他对于儿童文学的热爱愈发深厚, 并将其视作是毕生 的追求。这种主观层面的动机为他在儿童文学汉译事业中保持常青地位提供 了充足动力。据悉, 任溶溶老师通晓俄、英、意、日四国语言, 并向中国译 介了包括《安徒生童话全集》、《木偶奇遇记》、《吹小号的天鹅》、《夏洛的 网》等在内的三百余种外国儿童文学名著[13]。因任溶溶老师的大部分译本都 旨在通过滑稽有趣的故事来反映社会现实, 既达到娱乐儿童读者的目的, 又 能够通过阅读来帮助儿童明晰生活哲理, 树立正确的三观, 教育内涵丰富。

生态翻译环境可操纵译文的选择, 因此任溶溶老师选择翻译的文本具有 很强的时代特色。他通常借助于诙谐幽默的写作方式来反映深刻的社会现实, 使得读者在情感上得到满足, 同时也通过故事的情节讲述来不断引导儿童正 确三观的树立, 帮助其茁壮成长 [14]。在翻译过程中, 不可避免地会面临社会 条件与形势的变化, 单一的翻译策略并不能满足时代变迁的需求。因此, 他 根据实际情况, 选择合适的文本, 并适当调整翻译策略, 因此为其译作在儿 童文学汉译领域坐拥不可撼动的地位提供了充分的客观条件 [15]。

\section{2. 生态翻译学理论简述}

首先, 与传统翻译理论不同, 生态翻译学理论强调 “译有所为” 指导下 的 “译者中心性” 的重要意义。“译有所为” 或 “以译行事” 既可以是翻译 行为的动因, 也可以是翻译活动的效果 [8]。通俗来说, “译有所为” 主要体 现在下述两个方面: 一方面, 指的是译者从事翻译尤其特定的动因, 是主观 层面的倾向与选择; 另一方面, 则是翻译出来的东西可以成为有用之物, 是 客观层面的评价与效果 [7]。此外, 生态翻译学关注译者的作用与地位, 认为 译者有责任去努力适应生态翻译环境, 致力于构建并培育译语生态, 关注译 品推介的特征与反馈, 力求译品能够在译语生态中长存 [16]。可以说, 生态翻 译学 “译者中心” 论断的出现, 解构了传统观念下 “原文中心” 或 “译文中 心” 的极端性, 构成了 “原文 - 译者 - 译文” 三者互联互动的有机整体。有 
部分研究将 “译者中心性”与 “译者主体性”、“译者主导作用” 混为一谈。 但 “译者为中心” 并不代表译者能够为所欲为, 而是要受到 “适者生存”、

“事后追惩” 法则以及 “翻译生态环境” 的选择和制约。

而生态翻译学是在 “翻译适应选择论” 研究的基础上发展起来的。该理 论将翻译过程与生物学进化过程进行类比, 并以达尔文生物进化论 “适应/选 择” 学说为指导, 指出翻译是 “译者适应翻译生态环境的选择活动” [5]。翻 译过程中要适当进行 “选择性适应” 与 “适应性选择”, 强调 “适应” 与 “选 择” 的交互作用。前者代表的是译者在翻译过程中首先要 “适应生态环境” ; 后者则代表译者在适应的基础上, 根据翻译生态环境来决定对译文的选择。 这两者互为表里, 相互制约, 只有 “整合适应选择度” 最高的翻译, 才有可 能成为最佳的翻译 [7]。2001 年, 胡庚申教授首次提出 “翻译生态环境” 这一 概念。近年来, 有关这一概念的界定也在随着实践不断深化推进, 其概念外 延也在不断的调试和扩大。具体来说, 生态翻译环境可视作是除译者以外的 一切构成因素: 既可从宏观上考察国家社会政治制度和语言政策, 也可从微 观上衡量翻译研究本体的内部构成; 既可从物质环境入手, 也可从精神环境 入手[17]。对于儿童文学翻译来说, 它受到特定社会背景和国家语言政策的影 响, 同时也会受到儿童认知阶段性的影响。因此, 儿童文学翻译要从受众可 接受的范围去考虑翻译文本、翻译策略等使用, 从而构成翻译生态环境的有 效联动。

\section{3. 基于生态翻译学 “三维转换” 翻译理论的译本解读}

一些推崇生态翻译学的学者[7] [14] [18]认为: 翻译方法可以依据 “三维” 转换的方式展开讨论及运用, 即译者首先要基于 “多维度适应与适应性选择” 的原则, 并在发挥自身主观能动性与创造性的基础之上, 相对地集中于语言 维、文化维和交际维的适应性选择转换, 根据自身需求与译作所期实现的效 果来选择适当的翻译策略与方法手段。至此, 下文将从 “三维转换” 视角切 入, 对《柳林风声》任译本进行评析解读。

\subsection{1. 语言维: 语言信息的流动}

语言维侧重的是语言信息的流动与互通。译者在翻译过程中对语言维进 行适应性选择转换, 主要指的是翻译过程中对于语言形式的适应性选择转换 [7]。同时, 因语言具有层次性, 因此语言维涉及到的选择和转换可以从不同 层次上来考量。在具体的翻译过程中, 如何使得译作从最大程度上保证原作 的想要传达的含义, 做到忠实于原文, 也是十分重要的切入点。因此, 对于 译作者来说, 遣词造句需要经过精心设计方可呈现在读者面前。

*例 1:

【原文】So he scraped and scratched and scrabbled and scrooged, and then he scrooged again and scrabbled and scratched and scraped... (P1)

【任译】就这样, 他用他的小爪子忙着又是扒, 又是挖, 又是掘, 又是 抓, 接着又是抓, 又是掘, 又是挖, 又是扒……

在例 1 中, 主要考量的是四个词汇的翻译策略, 即: scraped (刮), scratched 
(抓), scrabbled (摸)和 scrooged; 任老师将其分别译作：扒、挖、掘、抓。不 同语言的使用者在进行阅读时有时候也需要调动不同的功能, 如编码或字词 识别的策略[19]。汉语是一种表意文字, 其字形具有独特的空间表征方式, 并 且与音节水平的语音表征存在联系; 而英语则是一种表音文字。原文中的四 个词汇均是由 “scr-” 前缀构成, 且出现了倒序性重复。任译本中, 译者有意 将这四个单词的汉译进行了再加工, 选择了四个同是提手旁 “未” 构词的汉 字, 与原文格式统一, 且在选词上尽可能贴近于原文的特点。但是关于 scrooge 这一单词的翻译, 本文持保留态度: 通过查阅相关资料可知, 该单词目前仅 保留着名词含义, 即 “吝啬鬼” ; 而任译本为何在此译为 “抓”, 未来研究 中需继续进行讨论。

*例 2:

【原文】 “There's cold chicken inside it," replied the Rat briefly; "cold tongue cold ham cold beef pickled gherkins salad French rolls cress sandwidges potted meat ginger beer lemonade sodawater...” (P5)

【任译】 “里面有冷鸡”, 河鼠简短地答道, “冷舌头、冷火腿、冷牛 肉、腌小黄瓜沙拉、法国面包卷、水芹三明治、罐头肉沙拉、汽水、柠檬汁、 苏打水…...”

例 2 发生的背景是河鼠在拿到装满午餐的柳条篮子之后, 好奇的鼠鼠满 怀期待地渴望从河鼠那里听到等候他们的丰盛午餐。原文没有采用标点符号, 想要体现的是河鼠对于午餐 “了如指掌”, 所以才能滔滔不绝地报出午餐的 名称。但是在任译本中, 增译了顿号作为不同餐食之间的连接, 虽然看起来 更加清晰直观, 但是读起来更像是生搬硬套、没有感情的复述, 缺少了原文 中的活泼特质。因此, 从语篇层面上来说, 该例与原文写作风格略有出入, 未能很好地体现出译文对于原文语境的适应与调整, 语言维的 “适应性选择” 表现较弱。

\subsection{2. 文化维: 文化内涵的沟通}

文化维侧重的是不同文化背景之下, 原文与译文之间文化内涵的交融与 沟通。译者在翻译过程中对文化维进行适应性选择转换, 主要指的是译者在 翻译过程中要关注源语文化和译语文化在性质和内容上的差异, 避免出现曲 解原文的现象 [7]。文化维度上的差异主要有两大来源: 一是中西方拥有不同 的文化背景, 二是中西方在选用语言时会基于不同的语境。因此, 译者在对 原文进行加工处理时, 是否有关照到双语之间存在的文化差异、注意到差异 后又将如何处理这些差异, 也是衡量与评价译本质量优劣的重要指征。

*例 3:

【原文】 The Mole had been working very hard all the morning, springcleaning his little home... Jumping off all his four legs at once, in the joy of living and the delight of spring without its cleaning, he pursued his way across the meadow till he reached the hedge on the further side. (P1-2)

【任译】一整个上午, 鼠鼠忙得不亦乐乎, 在他家那间小屋子里拼命地 大扫除……在生活的喜悦中, 在不用大扫除的春天欢乐中, 他同时用四条腿 
蹦跳起来, 一路跑过大草原, 一直来到远远那头的灌木树篱那里。

例 3 中，任老师将 “spring-cleaning” 译为 “大扫除”。在中国，大扫除 指的是为迎接新年的中国传统民俗之一; 而在西方国家, “spring-cleaning” 则指的是在春夏之际清扫杂乱的房屋, 以便迎接新开始。在这里, 没有能够 考虑到中西方文化的差异并进行标注, 所以在翻译下文中的 “spring without its cleaning” 时, 将其直译为 “不用大扫除的春天欢乐” , 读起来晦澀难懂, 且容易产生歧义。若能够在文中注明英汉之间对于该俗语的不同解读, 可能 在翻译时就能够避免采用午杂的语言结构, 同时也有助于读者的理解。在此, 可参考杨静远译本 [20]中的相关表述, 即 “生活的欢乐, 春天的愉悦, 又加上 免了大扫除的麻烦, 他乐得纵身一跳, 腾起四脚向前飞跑, 横穿草坪, 一直 跑到草坪尽头的篦笆前”。与此同时, 通过脚注的形式, 注明 “spring-cleaning” 在中西方语境下的内涵差异, 以便读者更好地了解其中蕴含的区别, 从而树 立良好的跨文化意识。

*例 4:

【原文】The Return of Ulysses

【任译】尤利西斯

例 4 选择的是该书第 12 章节的标题。该部分讲述的是河鼠、鼠鼠等主人 公发起奇袭的准备与过程, 以及夺回蛤蟆府邸之后重振家园、相聚一起举行 庆祝宴会的情形。作者在文末加以脚注写道: “尤利西斯是古希腊荷马史诗 《奥德修记》中的一位大英雄。”虽然很多读者并未能亲自读过《奥德修记》， 但是通过阅读脚注, 我们也能够明晰尤利西斯在其中充当的是一个正面角色。 虽然从某种程度上来说, 脚注为读者提供了可供参考的注释信息; 但是在翻 译时, 任译本未能直接将 “Return (归来)” 的含义译出, 如果没能第一时间 关注到脚注的信息, 则阅读这个标题时会感到十分突兀, 不利于读者很好地 把握语篇信息。

\subsection{3. 交际维：译者与读者的对话}

交际维侧重的是通过原文与译文的互动，基于译者与读者之间产生的“对 话” 与 “交际”。译者在翻译过程中对交际维进行适应性选择转换, 主要指 的是译者在翻译过程中关注双语交际意图的适应性选择转换 [7]。该维度以语 言维和文化维为基础, 是对这两者的延伸与扩展。翻译实则就是作者、译者 与读者之间互动的过程, 通俗来说, 译者通过 “自说自话” , 将原文作者想 要传达的信息加以加工处理后, 如数传递给读者。在此过程中, 三者形成了 一种动态平衡, 符合翻译生态环境的需求。对于儿童文学来说, 它面对的受 众群体主要是年幼的儿童。由于认知能力发展的限制, 他们对于文本的理解 能力还有所欠缺。因此, 儿童文学中所选用的语言、图像与页面排版均需满 足儿童的发展特征。如何能够创造出儿童喜闻乐见的文学作品、译介出符合 儿童年龄特性的文学作品, 也是在对儿童文学进行翻译时需要衡量的一个重 大因素。交际过程中需要涉及到大量的信息处理。因此, 翻译儿童文学作品 需要通过创造性地使用生动简练的语言表达, 来向儿童读者传递原文信息。 若使用晦澀难懂的学术性语言, 这对于小读者们来说, 无疑是一场巨大的认 
知灾难。因此, 从交际有效性的角度来选择翻译过程时, 需要时时刻刻顾及 儿童阅读需求。

倡导基于儿童本位论的译事过程与前文生态翻译学所倡导的 “译者中心 性” 并不矛盾。“译者中心” 仅仅指的是在翻译过程中, 译者需要根据自己 的理解对原文进行再创造, 而儿童本位论则表明, 不管是译事前、译事中还 是译事后, 都需要考虑到儿童的生理及心理特性, 从而在翻译时能够更好地 把握语言的运用, 体现出译者对于儿童读者的人文关怀。在翻译儿童文学作 品时, 译者需要时刻做到 “以儿童为本位”, 揣摩儿童读者的特点和接受水 平, 贯彻 “口语取向为本、童趣取向为本和情景化为本” 的原则。只有在此 背景下产出的译作才能更好地被儿童所接受[21]。任译本中常出现口语化的表 达, 对于语气词、感叹语句的处理可以称得上是惟妙惟肖, 如将 “I'm a mean beast” 译为 “我是个小气鬼”、将 “Lord!” 译为 “老天爷!”、将 “This has been a wonderful day!” 译为 “今天是个呱呱叫的好日子” 等, 都是基于中国 文化语境中的口语化、日常化表达, 更加贴近中国儿童的日常生活。

\section{3. 结语}

任溶溶老师是一个有情怀、心持热爱的儿童文学作家、翻译家。经过岁 月的洗礼与见证, 其译作流传至今, 成为陪伴众多中国儿童小读者成长的经 典之作。任老师在儿童文学翻译事业中能够时刻以儿童为本, 时刻从儿童所 需出发, 在翻译过程中以此为依据进行巧妙构思。因生态翻译学是集合了西 方生态学理论与东方哲学思想的一个新兴翻译理论, 是中国本土对于翻译理 论第一次创新性提出, 所以其有效性需要通过大量的论证与实践来证明。《柳 林风声》是一项基于自然生态的描写, 因此, 结合自然科学与人文科学 “生 态性” 的生态翻译学理论来对该译文进行评介未尝不是一种尝试与创新。

生态翻译学通过概念移植与跨学科类比尝试性建立一套翻译术语体系, 近年来得到了飞跃式发展 [22], 但是在明晰性、准确性和专业性的表现上欠缺。 该理论建立在 “翻译适应选择论” 的基础之上, 在外延不断扩展的过程中, 达尔文进化论所体现的自然科学方法能否移植于人文学科研究也是需要未来 进行深入讨论的一个话题。此外, 过多或不恰当地依赖于东方文化中的类比 思维, 也是后续研究需要规避的一个问题。同其他翻译理论一样, 生态翻译 学只能解决翻译中的某些问题, 而不能解释所有问题。因此, 作为中国翻译 学界的创新性与突破性成果, 生态翻译学的发展还要经过岁月的考证才能在 国际翻译学界中占据一席之地。此外, 对于任译本来说, 虽然大部分情况下 能够满足儿童的阅读需求, 但是在对一部分文本进行语言处理时, 仍呈现出 咒长复杂、䀲涩难懂的特征。此外, 该译本中缺少对于中西方文化差异上的 解释, 不利于儿童了解双语文化背景, 造成一定的阅读困难。该译本已问世 多年, 其经典性不言而喻。但是随着时代的发展, 文中的相关表述是否还能 够满足新一代儿童读者的需求, 后续又该如何进行修正, 也是未来需要思考 的一个问题。

综上所述, 本文在简述生态翻译学理论的基础上展开对《柳林风声》任 译本的评析, 一方面验证了生态翻译学理论应用于儿童文学翻译鉴赏的有效 
性, 另一方面则为分析译本提供了支持框架, 以期从中发现该译本的优势与 不足。但本文仍有一定的局限性。首先, 因文章篇幅的限制, 未能提供更多 的例句来验证生态翻译学理论的有效性, 对于译本的评析也较为单薄, 论证 的深度还不够。其次, 在评析译本的过程中, 仅结合某一例句做简要分析, 单纯地评述译本表达的 “好” 与 “坏” ，未能很好地结合不同的译本表达来 进行对比分析, 有较强的主观倾向, 不利于完整地呈现出译本的优势与不足。 最后, 本文虽能够在表述清楚理论的基础上完成译本鉴赏, 但缺少一定的创 新意识, 如: 未能为生态翻译学在儿童文学翻译中的有效性提供更为明确的 建议, 未能结合最新研究成果、丰富翻译实践等。这些不足也为后续生态翻 译学理论的发展、儿童文学翻译赏析进程提供了些许启示。

\section{基金项目}

中央高校基本科研业务费专项资金资助项目(项目编号：2021YJS194)。

\section{Conflicts of Interest}

The author declares no conflicts of interest.

\section{References}

[1] 应玲素. 论儿童文学与儿童阅读能力的培养[J]. 课程. 教材. 教法, 2006, 26(10): 53-57.

[2] 刀曼云. 现实中的 “乌托邦”一一儿童文学的功能及其应用 [J]. 语文建设, 2014(32): 36-37.

[3] 蒋风. 从“文化旅行”看现代儿童文学发展 [N]. 中国社会科学报, 2018-08-17(006).

[4] 胡庚申. 生态翻译学: 产生的背景与发展的基础[J]. 外语研究, 2010(4): 62-67.

[5] 胡庚申. 生态翻译学解读[J]. 中国翻译, 2008, 29(6): 11-15.

[6] 胡庚申. 新生态主义与新生态主义翻译观[J]. 鄱阳湖学刊, 2019(6): 5-11.

[7] 胡庚申. 生态翻译学的研究焦点与理论视角 [J]. 中国翻译, 2011, 32(2): 5-9.

[8] 思创.哈格斯. 生态翻译学的国际化进展与趋势 [J]. 上海翻译, 2013(4): 1-4, 20.

[9] 任溶溶. 柳林风声 [M]. 北京: 光明日报社, 2015.

[10] 舒伟. 论《柳林风声》作者的人生感悟与童话叙事的关联[J]. 解放军外国语学院 学报, 2012, 35(1): 91-95.

[11] 舒伟. 论《柳林风声》的经典性儿童文学因素[J]. 贵州社会科学, 2011(12): 16-19.

[12] 孙悦. 一个快乐的“老小孩”任溶溶[J]. 编辑学刊, 2009(2): 48-52.

[13] 荆素蓉, 米树江. 儿童文学翻译家任溶溶研究综述[J]. 外国语文研究, 2015(5): 70-75.

[14] 王爱琴, 陈光明. 任溶溶儿童文学翻译的生态翻译学诠释[J]. 安庆师范学院学报: 社会科学版, 2012, 31(3): 27-30.

[15] 张嘉坤, 李新新. 任溶溶儿童文学翻译研究综述 [J]. 海外英语(上), 2016(12): 128-129.

[16] 胡庚申. 从“译者中心”到“译者责任”[J]. 中国翻译, 2014, 35(1): 29-35,126.

[17] 胡庚申. 对生态翻译学几个问题“商榷”的回应与建议[J]. 中国翻译, 2014, 35(6): 86-89.

[18］李荣荣, 邵华. 生态翻译学的研究焦点[J]. 海外英语(下), 2019(1): 35-36. 
[19] OECD (Organization for Economic Co-Operation and Development) (2007) Understanding the Brain: The Birth of a Learning Science. Organization for Economic Co-Operation and Development, Paris.

[20] 杨静远. 柳林风声 [M]. 沈阳: 春风文艺出版社, 2015.

[21] 刘秋喜. 基于儿童读者的儿童文学翻译[J]. 海外英语(上), 2016(10): 113-114.

[22] 张小丽. 关于生态翻译学理论建构的三点思考[J]. 浙江师范大学学报: 社会科学 版, 2015, 40(6): 73-78.

\section{Appendix (Abstract and Keywords in Chinese) 生态翻译学视阈下儿童文学经典译作评介 -一以《柳林风声》任译版为例}

摘要: 较西方儿童文学相比, 我国本土儿童文学的发展起步较晚, 目前仍存 在原创性不足、国际化程度较低等问题。为了丰富儿童的阅读体验, 促进其 阅读能力乃至未来学业能力发展, 引介外国儿童文学作品是一条重要途径。 在引介过程中, 翻译充当着嫁接原文和译文的桥梁。目前, 国内有关儿童文 学译本评析的文献主要基于接受美学理论、功能对等等传统翻译理论展开论 述, 将生态翻译学理论纳入儿童文学经典译作评价的研究较为缺乏。因此, 本文致力于梳理生态翻译学相关理论及研究焦点, 并以此为理论指导对任溶 溶版《柳林风声》汉译本进行评介: 一方面, 旨在通过生态翻译学来检验并 评析任译本; 另一方面, 则对生态翻译学在儿童文学作品翻译中所发挥的效 力及作用进行分析。

关键词: 生态翻译学, 儿童文学, 《柳林风声》, 英译汉 